\title{
A ESPIROMETRIA DE INCETIVO NO DESEMPENHO DE ATLETAS
}

\section{SPIROMETRY OF INCEPTION IN THE PERFORMANCE OF ATHLETES}

Flávia Rocha Santos

Acadêmica do $9^{\circ}$ período do Curso de Fisioterapia da UNIPAC. flaviaroch25@gmail.com

Matteus Cordeiro de Sá

Especialista em Fisioterapia Traumato-ortopédica . Curso de Fisioterapia da UNIPAC matteuscordeirodesa@gmail.com

\section{Walace Coelho da Silva}

Acadêmica do $9^{\circ}$ período do Curso de Fisioterapia UNIPAC

wcoelho21@hotmail.com

\section{Resumo}

Objetivo: O objetivo desta pesquisa foi mostrar como a Fisioterapia Desportiva Preventiva associada a recursos da Fisioterapia Respiratória tem a capacidade de aumentar o rendimento técnico, físico e ainda diminuir o risco de lesões em atletas. Metodologia: Para a elaboração deste trabalho foi realizado a coleta de dados por meio da revisão de literatura. Foram utilizados instrumentos de pesquisa bancos de dados virtuais como Google Acadêmico, Scielo, Lilacs, PEdro. O referencial literário aqui descrito foi pesquisado através artigos científicos em português e em inglês, buscadores on-line, revistas cientificas e obras bibliográficas relacionadas ao assunto. A Espirometria de Incentivo no Desempenho de Atletas: O espirômetro de incentivo (EI) é um recurso que promove altos volumes pulmonares e sustenta inspirações máximas levando ao aumento do volume corrente, do tempo inspiratório e do fluxo inspiratório que assegura a estabilidade alveolar. Existem diferentes tipos de aparelhos que são volume ou fluxo-dependente. $\mathrm{O}$ espirômetro pode ser utilizado tanto na fisioterapia respiratória quanto na esportiva com o intuito de auxiliar na melhora da função pulmonar durante competições e exercícios intensos. Conclusão: Tendo em vista essa problemática, torna-se necessário que haja mais pesquisas acerca do Treinamento Muscular Inspiratório, e que sejam produzidas e propagadas nacionalmente pelos fisioterapeutas para que cada vez mais pessoas 
(atletas profissionais ou não) possam se beneficiar vastamente do treinamento muscular inspiratório durante o processo de preparação física ou até mesmo reabilitacional, oportunizando lhe mais desempenho à sua prática desportiva.

Palavras-chave: espirometria; fisioterapia respiratória; desempenho de atletas.

\begin{abstract}
Objective: The objective of this research was to show how the Preventive Sports Physiotherapy associated with Respiratory Physical Therapy resources has the capacity to increase the technical and physical performance and also to reduce the risk of injuries in athletes. Methodology: For the preparation of this work, the data collection was performed through the literature review. We used research tools such as Google Scholar, Scielo, Lilacs, PEdro. The literary reference described here was searched through scientific articles in Portuguese and English, online search engines, scientific journals and bibliographical works related to the subject. Incentive Spirometry in Athlete Performance: The incentive spirometer $(\mathrm{EI})$ is a feature that promotes high lung volumes and sustains maximal inspirations leading to increased tidal volume, inspiratory time, and inspiratory flow that ensures alveolar stability. There are different types of appliances that are volume or flow-dependent. The spirometer can be used in both respiratory and sports physiotherapy with the aim of helping to improve lung function during intense competitions and exercises. Conclusion: In view of this problem, there is a need for more research on Inspiratory Muscular Training, and to be produced and propagated nationally by physiotherapists so that more and more people (professional and non-professional athletes) can benefit vastly from muscle training inspiratory during the process of physical preparation or even rehabilitation, giving you more performance to your sport.
\end{abstract}

Keywords: spirometry; respiratory fisioterapy; performance of athletes.

\title{
Introdução
}

A maioria das pessoas associa a fisioterapia somente aqueles exercícios necessários após lesões musculares, cirurgias ou recomendados para pessoas idosas. Mas, na verdade, a área é muito mais ampla e capaz de atender diversos quadros, sejam eles para tratar condições físicas pontuais ou persistentes. Por se tratar de uma área ampla, as especialidades são diversas (Luiza, 2018).

A fisioterapia respiratória visa melhorar a dinâmica respiratória e a distribuição do ar inalado pelo pulmão [...]. São aplicadas técnicas manuais, como também aparelhos que ajudam na aplicação deste tipo de fisioterapia (Sautil, 2014). 
A fisioterapia tem como objetivo tratar os indivíduos portadores de um quadro patológico e restabelecer a funcionalidade perdida em decorrência deste quadro. Dessa forma, atua-se também na prevenção, associada à potencialização máxima das funções do atleta e à orientações de treinamento, que estão diretamente relacionadas ao desempenho, tornando clara a necessidade da atuação desse profissional dentro da equipe de treinamento desses indivíduos (Kurata, Junior, \& Nowtny, 2007).

O trabalho da fisioterapia esportiva é específico, pois todas as técnicas têm de ser aplicadas para a obtenção de resultados rápidos e funcionalmente efetivos, pois o atleta mais do que qualquer outro indivíduo precisará de executar todas as funções e o gesto técnico no máximo de potência e amplitude de movimentos (Oeiras, 2018).

Indicado para atletas amadores e praticantes regulares de esporte, melhorando o desempenho geral através do aumento da resistência dos músculos inspiratórios, aumento da capacidade de trabalho e redução da sensação de esforço e cansaço. Recomendado para atletas e praticantes de atividade física intensa, retardando a fadiga da musculatura respiratória, proporcionando melhora da performance (Rocha, 2012).

Os músculos respiratórios podem ser treinados para melhorar sua força e resistência, da mesma forma que os músculos esqueléticos de outras regiões do corpo. O desempenho do diafragma, conjuntamente com os outros músculos respiratórios, tem implicações diretas para a manutenção de uma ventilação pulmonar fisiológica e é determinante na qualidade da função ventilatória (Rocha, 2012).

A fisioterapia respiratória aplicada de forma preventiva, é de grande valia para o treinamento de atletas pré-competições, através de técnicas associadas contribui positivamente para um bom desempenho. Então o treinamento muscular respiratório e a terapia de expansão pulmonar auxiliam no condicionamento físico, assim como, no aumento da resistência pulmonar. Tendo em vista que a espirometria de incentivo é um recurso de baixo custo e totalmente acessível, tornando-o um excelente método de preparação pré competição, potencializando os resultados, mostrando a eficácia da fisioterapia respiratória quando utilizada de forma preventiva, justifica-se o interesse neste estudo. $\mathrm{O}$ objetivo do presente trabalho foi estudar formas de como a Fisioterapia Respiratória pode ajudar no desempenho de atletas, técnicas e potencializadores. 
Para a elaboração deste trabalho foi realizado a coleta de dados por meio da revisão de literatura. Foram utilizados instrumentos de pesquisa bancos de dados virtuais como Google Acadêmico, Scielo, Lilacs, PEdro. O referencial literário aqui descrito foi pesquisado através artigos científicos em português e em inglês, buscadores on-line, revistas cientificas e obras bibliográficas relacionadas ao assunto.

Os critérios de inclusão adotados para a busca das obras foram referenciais literários publicados entre os anos de 2001 a 2018, com a finalidade de analisar o uso da espirometria de incentivo em atletas para melhora no condicionamento físico. Após o levantamento, realizouse leituras de cunho crítico que em seguida foram aplicados de acordo com os conhecimentos adquiridos.

\section{Espirometria}

A espirometria $($ do latim spirare $=$ respirar + metrum $=$ medida $)$ é a medida do ar que entra e sai dos pulmões. Pode ser realizada durante respiração lenta ou durante manobras expiratórias forçadas. A espirometria é um teste que auxilia na prevenção e permite o diagnóstico e a quantificação dos distúrbios ventilatórios. (Pereira, 2002).

Embora a espirometria consista em uma técnica de medidas respiratórias muito antiga é bastante empregada nos estudos sobre fisiologia respiratória, há indícios de seu surgimento, enquanto técnica de avaliação clínica, em meados do século XIX por meio de um trabalho pioneiro de Hutchinson, em 1846. Na década de 1940, com o acréscimo do quimógrafo e da cronometragem da capacidade vital forçada (CVF), Tiffeneau Gaensler contribuiu de forma decisiva para que o espirômetro saísse da bancada científica e fosse incorporado ao laboratório clínico (Costa \& Jamami, 2001).

A espirometria é um teste fisiológico que mede como um indivíduo inala ou exala volumes de ar em função de Tempo. O sinal primário medido na espirometria pode ser volume ou fluxo. A espirometria é inestimável como um teste de rastreio de saúde respiratória da mesma forma que a pressão arterial fornece informações importantes sobre a função cardiovascular saúde (MR Miller, et al., 2005). 


\section{Espirômetro de Incentivo}

A espirometria pode ser realizada com muitos tipos diferentes de equipamento, e requer cooperação entre o sujeito e examinador, e os resultados obtidos dependerão de fatores técnicos e pessoais (MR Miller, et al., 2005). Espirometria de incentivo também referida como maxi- inspiração imaterial, é realizada usando um dispositivo que fornece feedback quando o paciente inala em um fluxo ou volume extraído e sustenta a inflação por pelo menos 5 segundos (Ruben D Restrepo, et al., 2011).

Os equipamentos utilizados na espirometria são de variados tipos e marcas e, com os avanços tecnológicos, a cada dia surgem novos modelos. [...] Todos os espirômetros devem fornecer informações sobre capacidade, exatidão da acurácia, linearidade, durabilidade e débito (Costa \& Jamami, 2001).

Dentre os mais antigos estão os espirômetros d'água, selados em água, ou com sistemas de roldanas, compensados com peso. Há um espirômetro do tipo pistão, que funciona com base no deslocamento horizontal de um pistão, possibilitando que o ar desloque-o gerando registros gráficos das medidas ventilatórias. [...] Os espirômetros mais modernos são construídos a base de sensores de fluxo, dentre os quais estão os de pressão diferencial (pneumotacógrafos ou pneumotacômetros), os termístores, os turbinômetros e os ultra-sônicos (Costa \& Jamami, 2001).

Os EI alineares podem ser orientados a fluxo ou a volume, de acordo com o padrão de ativação. Em vista disso, alguns estudos sugerem maior benefício da orientação a volume por ser mais fisiológica, uma vez que o volume de treinamento é constante até atingir a capacidade inspiratória máxima ou um nível pré-determinado em volume pelo terapeuta; e o fluxo é laminar, característico de vias aéreas de pequeno calibre, o que proporciona um menor trabalho respiratório imposto. Independente do modelo os objetivos são comuns, tais como: incentivar a inspiração máxima sustentada, proporcionar a reexpansão pulmonar, aumentar a permeabilidade das vias aéreas e também fortalecer os músculos respiratórios, devido a capacidade de gerar sobrecarga muscular (REIS, I M M, et al., 2015).

O paciente é instruído a segurar o espirômetro na posição vertical, expire normalmente e, em seguida, coloque o lábios firmemente ao redor do bocal. O próximo passo é um lento 
inalação para levantar a bola (orientada pelo fluxo) ou o pistão/placa (orientada por volume) na câmara para o alvo definido. No inalação máxima, o bocal é removido, seguido por uma expiração normal e expiração normal (Ruben D Restrepo, et al., 2011).

\section{Treinamento Muscular Inspiratório}

O TMI é um método de aplicação de uma carga externa quantificável aos músculos inspiratórios, sendo a pressão desenvolvida pelos músculos inspiratórios que impulsiona a ventilação. Os músculos inspiratórios, incluindo o diafragma, são morfológica e funcionalmente músculos esqueléticos e, portanto, devem responder ao treino da mesma forma que qualquer músculo locomotor, se for aplicada uma carga fisiológica apropriada. (Fábio Esteves, et al., 2016).

Os músculos respondem ao treino, melhorando principalmente a sua força, velocidade de contração, potência, endurance ou qualquer outra combinação que advenha destas. Dado que os músculos respondem sob variadas formas específicas, mediante o estímulo de treino, compreende-se que diferentes regimes de treino induzem ligeiras alterações em cada característica fisiológica (Fábio Esteves, et al., 2016).

\section{A Espirometria de Incentivo no Desempenho de Atletas}

Analisando o contexto de espirometria, segundo Santos, et al. (2012) a espirometria é um teste escolhido para avaliação funcional de pessoas com distúrbios pulmonares, sendo realizada pré ou pós uso de broncodilatadores. De acordo com Durmic, et al. (2015) a espirometria é um teste de função pulmonar referência que mede como um indivíduo inspira ou expira volumes de ar em função do tempo. É o mais importante e mais comum teste de função pulmonar, que se tornou imprescindível para a prevenção, o diagnóstico e a avaliação de diversas deficiências respiratórias.

Segundo Pereira (2002) a espirometria é um teste que auxilia na prevenção e permite o diagnóstico e a quantificação dos distúrbios ventilatórios. A espirometria deve ser parte integrante da avaliação de pacientes com sintomas respiratórios ou doença respiratória 
conhecida. Nesse sentido, a espirometria pode ser utilizada na preparação de atletas com o intuito de fortalecer a musculatura inspiratória como também melhorar a complacência pulmonar.

O espirômetro de incentivo (EI) é um recurso que promove altos volumes pulmonares e sustenta inspirações máximas levando ao aumento do volume corrente, do tempo inspiratório e do fluxo inspiratório que assegura a estabilidade alveolar. Existem diferentes tipos de aparelhos que são volume ou fluxo-dependente. Alguns estudos têm demonstrado diferentes comportamentos dos EI disponíveis, principalmente no que diz respeito ao ganho de volumes pulmonares e ao trabalho respiratório imposto (Diniz, et al., 2011). De modo que o EI auxilia na manutenção do padrão respiratório durante as competições.

De acordo Marques \& Faria (2009) os espirômetro de incentivo (EI) são instrumentos que fornecem feedback visual ou auditivo, encorajando os pacientes a realizarem inspirações máximas sustentadas. Inspirações profundas levam ao aumento da pressão transpulmonar e, associadas à pausa inspiratória, promovem insuflação e recrutamento alveolar, contribuindo para a estabilização dos alvéolos, melhorando a complacência e ventilação alveolar. Os EI são utilizados com o objetivo de restaurar os volumes pulmonares, modificando o padrão respiratório e de ventilação pulmonar. Desta forma, fica claro como o EI interfere diretamente na capacidade pulmonar do atleta. Visto que haverá um aumento da ventilação pulmonar gerando uma melhora a respiração durante a prática de esportes.

Durmic, et al. (2015) demonstrou que é possível distinguir os atletas da população geral, pois aqueles geralmente apresentam melhor função cardiovascular, maior volume sistólico e maior débito cardíaco máximo. Portanto, poderia supor que os atletas apresentam valores espirométricos maiores que os observados na população geral. Além disso, é possível que atletas altamente treinados apresentem alterações mal adaptativas do sistema respiratório, tais como obstrução intratorácica e extratorácica, limitação do fluxo expiratório, fadiga muscular respiratória e hipoxemia induzida pelo exercício, que podem influenciar seu desempenho.

Neste sentido, segundo Silva, et al. (2018) o treinamento muscular inspiratório (TMI) consiste em um método que vem sendo investigado como alternativa, para que através de seu uso os atletas possam obter benefícios em suas performances. Esta intervenção vem sendo sugerida como um programa de treinamento que pode aumentar o desempenho em atletas e 
indivíduos saudáveis. VENDRUSCULO \& DONADIO (2015) diz que o TMI pode ser uma forma de evitar a fadiga muscular inspiratória, retardar a dispneia, melhorando a capacidade ventilatória, durante o exercício e a qualidade de vida.

\section{Considerações Finais}

Há uma necessidade imediata da ascensão da Fisioterapia como uma ciência baseada em evidências e analisada fortemente em referenciais científicos validados. Observa-se que especificamente a área das ciências do esporte, nos últimos anos foram responsáveis por uma intensa produção científica na qual o Treinamento Muscular Inspiratório aplicados à maioria das modalidades esportivas foram temas amplamente pesquisados e fragmentados, atualmente sendo reconhecidos como peças fundamentais e essenciais a qualquer programa com objetivo de aumentar o desempenho geral do atleta, onde existem estudos cientificamente embasados. algum tempo, entretanto, lamentavelmente essa ainda não parece ser a realidade

Muitos dos autores citados acima, têm defendido a sua ampla valorização e emprego há da maioria dos centros de reabilitação e academias. Tendo em vista essa problemática, torna-se necessário que haja mais pesquisas acerca do Treinamento Muscular Inspiratório, e que sejam produzidas e propagadas nacionalmente pelos fisioterapeutas para que cada vez mais pessoas (atletas profissionais ou não) possam se beneficiar vastamente do treinamento muscular inspiratório durante o processo de preparação física ou até mesmo reabilitacional, oportunizando lhe mais desempenho à sua prática desportiva.

\section{Referências}

Luiza, A. (2018). O que é Fisioterapia: respiratória, esportiva, dermatofuncional e mais. Fonte: minutosaudavel.com.br. Disponível em:

$<$ https://minutosaudavel.com.br/fisioterapia/>. Acesso em 08 de Dezembro de 2018.

Sautil. (2014). Fisioterapia respiratória. Fonte: sautil.com.br. Disponível em $<$ http://www.sautil.com.br/saude-para-voce/sauderespiratoria/conteudo/fisioterapiarespiratoria>. Acesso em 01 de Dezembro de 2018. 
Kurata, D. M., Junior, j. M., \& Nowtny, J. P. (2007). INCIDÊNCIA DE LESÕES EM ATLETAS PRATICANTES DE FUTSAL. Iniciação Científica CESUMAR, 45-51. Fonte: periodicos.unesc.net. Disponível em:

$<$ http://periodicos.unesc.net/saude/article/view/6>. Acesso em 25 de Maio de 2019.

Oeiras, F. (2018). Fisioterapia Desportiva. Fonte: fisioterapiaoeiras.com. Disponível em: <https://www.fisioterapiaoeiras.com/servicos/fisioterapia/fisioterapia-desportiva $>$. Acesso em 08 de Dezembro de 2018.

Rocha, M. R. (2012). Treinamento muscular respiratório. Fonte: Atividade Corporal. Disponível em: <http://blogs.viaeptv.com/blogs/atividadecorporal/tag/inspirometrosdeincentivo/>. Acesso em 08 de Dezembro de 2018.

Costa, D., e Jamami, M. (2001). BASES FUNDAMENTAIS DA ESPIROMETRIA. Revista brasileira fisioterapia, 95-102. Fonte: Associação Brasileira de Fisioterapia. Disponível em: <http://www.ufjf.br/nfbio/files/2016/06/Espirometria-2.pdf>. Acesso em 18 de Maio de 2019.

MR Miller, J. Hankinson, V. Brusasco, F. Burgos, R. Casaburi, A. Coates, . . . J. Wanger.

(2005). Standardisation of spirometry. EUROPEAN RESPIRATORY JOURNAL, 319338.

Fonte: erj.ersjournals.com. Disponível em:

<https://erj.ersjournals.com/content/erj/26/2/319.full.pdf>. Acesso em 25 de Maio de 2019.

Ruben D Restrepo, Richard Wettstein, Leo Wittnebel, \& Michael Tracy. (2011). Incentive Spirometry. RESPIRATORY CARE, 1600-1604. Fonte: AARC CLINICAL PRACTICE GUIDELINE: INCENTIVESPIROMETRY. Disponível em:

$<$ http://rc.rcjournal.com/content/respcare/56/10/1600.full.pdf>. Acesso em 25 de Maio de 2019.

REIS, I M M, PESSOA-SANTOS, B V, BASSO-VANELLI, R P, DI LORENZO, V A P, \& JAMAMI, M. (2015). Efeitos do treinamento com espirômetros de incentivo a fluxo e a volume em indivíduos saudáveis. Revista Brasileira de Ciência e Desenvolvimento, 104-112.

Fonte: http://docs.bvsalud.org. Disponível em:

<http://docs.bvsalud.org/biblioref/2017/05/833714/efeitos-do-treinamentocomespirometros.pdf>. Acesso em 25 de Maio de 2019. 
Fábio Esteves, Inês Santos, João Valeriano, \& M. Teresa Tomás. (2016). Treino de músculos inspiratórios em indivíduos saudáveis: estudo randomizado controlado. SAÚDE \& TECNOLOGIA, 5-11. Fonte: Saude e Tecnologia: revista científica. Disponível em: <https://web.estesl.ipl.pt/ojs/index.php/ST/article/view/1331>. Acesso em 25 de Maio de 2019.

Santos, J. S., Jr, G. A., Bliacheriene, A. C., \& Foster, A. C. (2012). Protocolos Clínicos e de Regulação. Rio de Janeiro: ELSEVIER. Fonte: edisciplinas.usp.br. Disponível em: $<$ https://edisciplinas.usp.br/pluginfile.php/4528654/mod_resource/content/1/Protocolos $\% 20 \mathrm{cl}$

\%C3\%ADnico\%20e\%20de\%20regula\%C3\%A7\%C3\%A3o\%20-

$\% 20$ Acesso\%20\%C3\%A1\%20rede\%20de\%20sa\%C3\%BAde.pdf $>$. Acesso em 25 de Maio de 2019.

Durmic, T., Lazovic, B., Djelic, M., Lazic, J. S., Zikic, D., Zugic, V., . . Mazic, S. (2015). Influências específicas dos esporte nos padrões respiratórios em atletas de elite. Fonte: scielo.br. Disponível em:

<http://www.scielo.br/pdf/jbpneu/v41n6/pt_1806-3713jbpneu-41-06-00516.pdf >. Acesso em 10 de Dezembro de 2018.

Pereira, C. A. (2002). Espirometria. J Pneumol, 1-79. Fonte: http://www.saude.ufpr.br. Disponível em:

<http://www.saude.ufpr.br/portal/labsim/wpcontent/uploads/sites/23/2016/07/Suple_13 9_45_11-Espirometria.pdf>. Acesso em 18 de Maio de 2019.

Diniz, G. d., Souza, L. T., Martins, M. d., Costa , R. P., Figueiredo, P. H., \& Pessoa, B. P. (2011). Efeitos da utilização do espirômetro de incentivo com três diferentes interfaces em pacientes submetidos à cirurgias torácicas e abdominais altas. ASSOBRAFIR Ciência, 39-47.

Fonte: http://www.uel.br. Disponível em:

<http://www.uel.br/revistas/uel/index.php/rebrafis/article/viewFile/7869/8156>. Acesso em 18 de Maio de 2019.

Marques, C. d., \& Faria, I. d. (2009). TERAPIA INCENTIVADORA DA INSPIRAÇÃO: UMA REVISÃO DAS TÉCNICAS DE ESPIROMETRIA DE INCENTIVO A FLUXO E A VOLUME E O BREATHSTACKING. Fonte: Revista Brasileira em Promoção da Saúde. Disponível em: <http://periodicos.unifor.br/RBPS/article/view/367/2250>. Acesso em $1^{\circ}$ de Dezembro de 2018. 
Silva, H. P., Moura, T. S., \& Silveira, F. d. (2018). EFEITOS DO TREINAMENTO MUSCULAR INSPIRATÓRIO EM ATLETAS DE FUTEBOL. Fonte: Revista brasileira de Prescrição e Fisiologia do Exercício. Disponível em:

$<$ http://www.rbpfex.com.br/index.php/rbpfex/article/view/1468>. Acesso em 10 de Dezemro de 2018.

VENDRUSCULO, F. M., \& DONADIO, M. V. (2015). Efeitos do treinamento muscular inspiratório em pacientes com fibrose cística. ASSOBRAFIR Ciência, 33-41. Fonte: http://www.uel.br. Disponível em: 〈http://www.uel.br/revistas/uel/index.php/rebrafis/article/viewFile/20113/16987>. Acesso em 30 de Maio de 2019. 